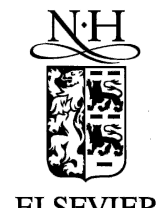

Pattern Recognition Letters 19 (1998) 439-446

Pattern Recognition

Letters

ELSEVIER

\title{
Window-size determination for granulometrical structural texture classification $^{1}$
}

\author{
Sen-Ren Jan, Yuang-Cheh Hsueh * \\ Department of Computer and Information Science, National Chiao-Tung University, Hsinchu, Taiwan 300
}

Received 11 September 1997; revised 21 January 1998; accepted 22 January 1998

\begin{abstract}
In this paper we present a method to predict the window size when determining the local granulometry for a structural texture image set. The proposed method is based on the concept of periodicity property of structural texture images. It suggests that one may choose the minimum odd number not less than the maximum periods of texture images as a window size. (C) 1998 Elsevier Science B.V. All rights reserved.
\end{abstract}

Keywords: Granulometry; Local granulometry size distribution; Window size; Structural texture; Texture periodicity; Covariance; Co-occurrence matrix

\section{Introduction}

Dougherty and his colleagues introduce the concept of local granulometric size distributions and apply it to image segmentation (Dougherty et al., 1989) and pixel classification (Dougherty et al., 1992a). Their works indicate that moments of local granulometric size distributions provide good feature sets for pixel classification, however, a feature value from a local granulometric size distribution is a good descriptor of an image only when an effective window size is chosen.

A good result of the pixel classification is obtained only when the chosen window contains suffi-

\footnotetext{
* Corresponding author. E-mail: ychsueh@cis.nctu.edu.tw.

${ }^{1}$ This work was partially supported by the National Science Council of the R.O.C., under Grant NSC 84-2112-M-009-038.
}

cient information. Thus, the selection of an effective window size is very important. For a particular grain model, there is an inverse relation between the variation of the PSM (pattern spectrum mean) and the window size (Dougherty et al., 1992b). In this paper, we propose a method to predict a window size for a structural texture image set. A structural texture image possesses a property called texture periodicity. By investigating texture periodicities of the given images, we can determine the window size that in turn determines the local granulometry. The proposed method is based on the co-occurrence matrix (for the binary case) and the covariance calculation (for the greyscale case). We first find the texture periodicity and then determine a reasonable window size.

The rest of this paper is organized as follows. In Section 2, we briefly review morphological granulometries and local granulometries. In Section 3, we 
review the notions of co-occurrence matrices and covariance that will be used in the present study. In Section 4, we describe the proposed method in detail. In Section 5, we show experimental results and give a discussions. Finally, we draw some conclusions in Section 6.

\section{Granulometry and local size distribution}

Let $S$ be a bounded set and $E_{0}, E_{1}, E_{2}, \ldots$ an increasing sequence of structuring elements such that $E_{k+1}$ is $E_{k}$-open, $k=1,2, \ldots$ Then the opened images $S \circ E_{0}, S \circ E_{1}, S \circ E_{2}, \ldots$ form a decreasing sequence called a granulometry (Matheron, 1975). Moreover, a decreasing function $\Omega(k)$ can be obtained by counting the number of pixels remaining in each succeeding opening. Since $S$ is bounded, $\Omega(k)$ $=0$ for sufficiently large $k$. If $E_{0}$ consists of a single pixel, then $\Omega(0)$ gives the image area of $S$ itself. The function $\Omega(k)$ is called a size distribution. The normalization $\Phi(k)=1-\Omega(k) / \Omega(0)$ is a probability distribution function and the discrete derivative $\mathrm{d} \Phi(k)=\Phi(k+1)-\Phi(k)$ is a probability mass function.

Local granulometric size distributions, introduced by Dougherty et al. (1989), are variations of size distributions alluded to the above. As in the original granulometry, the entire image is opened successively by a sequence of increasing structuring elements. Then, by placing a window at each pixel $x$ in the image and by taking a pixel-count in the window at each stage of the granulometry, a local granulometric size distribution $\Omega_{x}(k)$ is obtained for $x$. Then each size distribution is normalized and differentiated to generate the local pattern spectrum $\mathrm{d} \Phi_{x}(k)$.

Consider the model in (Dougherty et al., 1992b). Let the regions $S_{r}$ and $S_{R}$ partition an image $S$ such that they consist of disjoint balls of radius $r$ and $R$, respectively, where $r<R$. The proportion of the number of balls in each region to the region area is assumed to be a constant $q$. Now suppose $W$ is the window determining the local granulometric size distribution for a pixel $x$. In the case when $W$ lies partially in both $S_{r}$ and $S_{R}$, the area of $W \cap S_{r}$ and $W \cap S_{R}$ are assumed to be $p W$ and $(1-p) W$, re- spectively, where $0<p<1$. Moreover, no balls are assumed to intersect the boundary of $W$. Then

$\Omega_{x}(t)= \begin{cases}q \pi W\left[p r^{2}+(1-p) R^{2}\right] & \text { for } t \leq r, \\ q \pi W(1-p) R^{2} & \text { for } r<t \leq R, \\ 0 & \text { for } R<t .\end{cases}$

Normalization and differentiation yields:

$$
\begin{aligned}
\mathrm{d} \Phi_{x}(t)= & {\left[1-\frac{(1-p) R^{2}}{p r^{2}+(1-p) R^{2}}\right] \delta(t-r) } \\
& +\frac{(1-p) R^{2}}{p r^{2}+(1-p) R^{2}} \delta(t-R),
\end{aligned}
$$

where $\delta(t)$ is the delta function. Thus,

$$
\begin{aligned}
\mu_{x} & =\frac{p r^{3}+(1-p) R^{3}}{p r^{2}+(1-p) R^{2}}, \\
\sigma_{x}^{2} & =\frac{p r^{4}+(1-p) R^{4}}{p r^{2}+(1-p) R^{2}}-\left(\frac{p r^{3}+(1-p) R^{3}}{p r^{2}+(1-p) R^{2}}\right)^{2} .
\end{aligned}
$$

Note that as $p \rightarrow 0$, we have $\mu_{x} \rightarrow R$ and $\sigma_{x} \rightarrow 0$, and as $p \rightarrow 1$, we have $\mu_{x} \rightarrow r$ and $\sigma_{x} \rightarrow 0$. Also note that $r<\mu_{x}<R$.

The density $\mathrm{d} \Phi_{x}$ can be used as a descriptor of the local texture at $x$, and the moments of the local granulometric size distributions can be used to classify pixels based on local texture. Interested readers can find more details in Dougherty et al. (1992a,b).

\section{Co-occurrence matrices and covariance}

In the current study, we are interested in choosing an effective window size that determines the local granulometries for a structural texture image set. In structural texture images, texture primitives are basic units to form the images. These can be found by using co-occurrence matrices (Zucker and Terzopoulos, 1980; Parkkinen et al., 1990; Starovoitov et al., 1995). A co-occurrence matrix $M$ consists of elements $M(i, j ; \mathrm{d} k, \mathrm{~d} l)$, each of which represents the number of times that a given feature $i$ occurs in a particular spatial relation $(\mathrm{d} k, \mathrm{~d} l)$ to another given feature $j$. Generally, $\chi^{2}$ statistics are used to measure the association between the grey values of the two pixels situated $(\mathrm{d} k, \mathrm{~d} l)$ apart. Let $M(i, j)$ denote 
the elements of the co-occurrence matrix and, $N$ the sum of all elements of $M(i, j)$. Here, for brevity, the displacement $(\mathrm{d} k, \mathrm{~d} l)$ is not explicitly given. The $\chi^{2}$ statistics are computed by the following formula (Parkkinen et al., 1990):

$\chi^{2}=\sum_{i=1}^{m} \sum_{j=1}^{m} \frac{\left(M(i, j)-\frac{M(i, \cdot) M(\cdot, j)}{N}\right)^{2}}{\frac{M(i, \cdot) M(\cdot, j)}{N}}$,

where $M(i, \cdot)$ and $M(\cdot, j)$ are row and column sums, and $m$ is the matrix size. The displacements $(\mathrm{d} k, \cdot)$ and $(\cdot, \mathrm{d} l)$ are used to analyse the horizontal and vertical periods, respectively. For the binary case, this method is fast and effective. However, it has to compute a large co-occurrence matrix for a grey scale image. In order to avoid the large co-occurrence matrix computation, we use the concept of covariance to find the texture primitives for grey scale images.

Let $S$ be an artificial texture image shown in Fig. 1(a), and let $B=\{0, \overrightarrow{1} h\}$ be a structuring element, where $\overrightarrow{1} h$ is parallel to the $x$-axis. Then the covariance of $S$ can be defined as $\operatorname{Cov}(h)=\operatorname{Mes}(S \ominus B)$, where $\operatorname{Mes}(A)$ denotes the number of black pixels in $A$ (Serra, 1982; Giardina and Dougherty, 1988). That is, the covariance $\operatorname{Cov}(h)$ is the measure of $S \ominus B$, the erosion of set $S$ by $B$. The calculated covariance is depicted in Fig. 1(b). The period of repetition can be found by investigating their spatial distribution (Serra, 1982) or by using convolution filters (Dewaele et al., 1988). In this study, we used a simple and intuitive approach. The width of the texture primitive is the distance between two adjacent local maxima (or minima). The height of the texture primi-

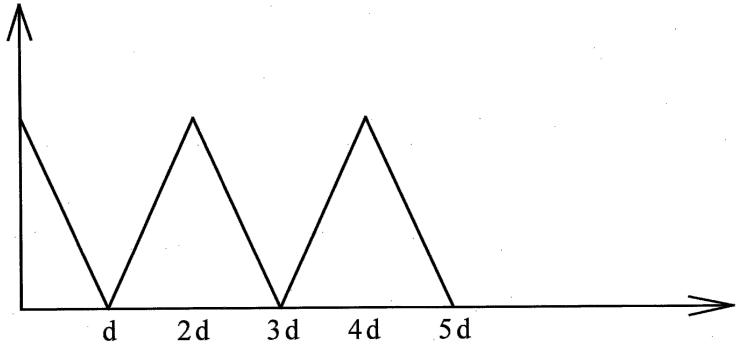

Fig. 2. Conditional covariance curve of Fig. 1(a).

tive can be similarly found by working along the $y$-axis.

The preceding results are easily generalized to grey scale images (Serra, 1982). For a grey scale image $S$, the covariance $\operatorname{Cov}(h)$ of $S$ is defined by

$$
\begin{aligned}
\operatorname{Cov}(h) & =E[[f(x)-p][f(x+h)-p]] \\
& =E[f(x) f(x+h)]-p^{2}
\end{aligned}
$$

where $f(x)$ denotes the grey value at point $x$ and $p=E[f(x)]$. Interested readers can find the details in Serra (1982).

In Fig. 1(b), we observe that the heights of the repetition peaks on the covariance curve are decreasing. To find the repetition period of a texture primitive, we define the conditional covariance $\operatorname{Cov}\left(\left.h\right|_{w}\right)$ to be $\operatorname{Mes}\left(S \cap w_{h}\right)$. Note that $w$ is a window selected from the texture image $S$. Instead of measuring the entire image $S, \operatorname{Cov}(h \mid w)$ just measures the area of the intersection of the shifted window $w_{h}$ and the image $S$. Fig. 2 exhibits the conditional covariance curve of the image in Fig. 1(a). Note that all peaks have equal height. Fig. 3(b) shows the conditional covariance curve of the texture image D102 in

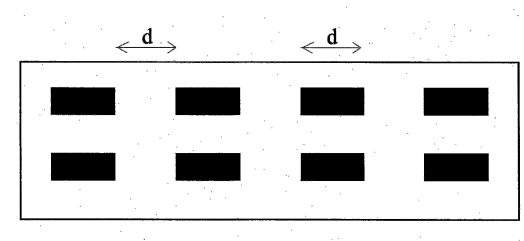

(a)

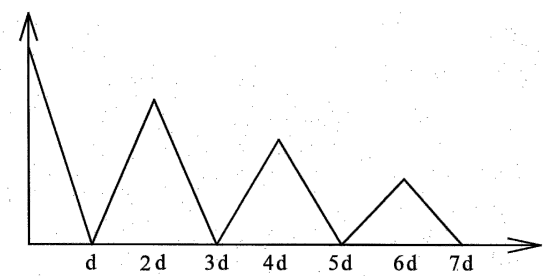

(b)

Fig. 1. (a) Artificial texture image; (b) covariance curve of (a). 
(a)

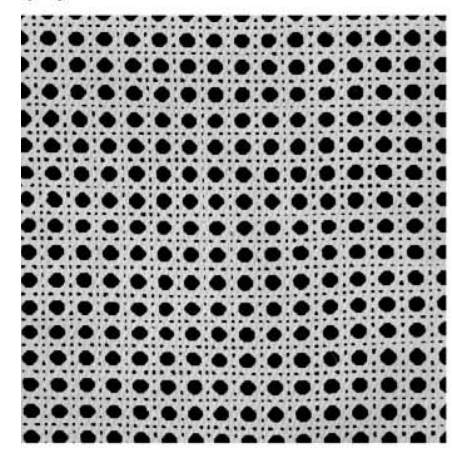

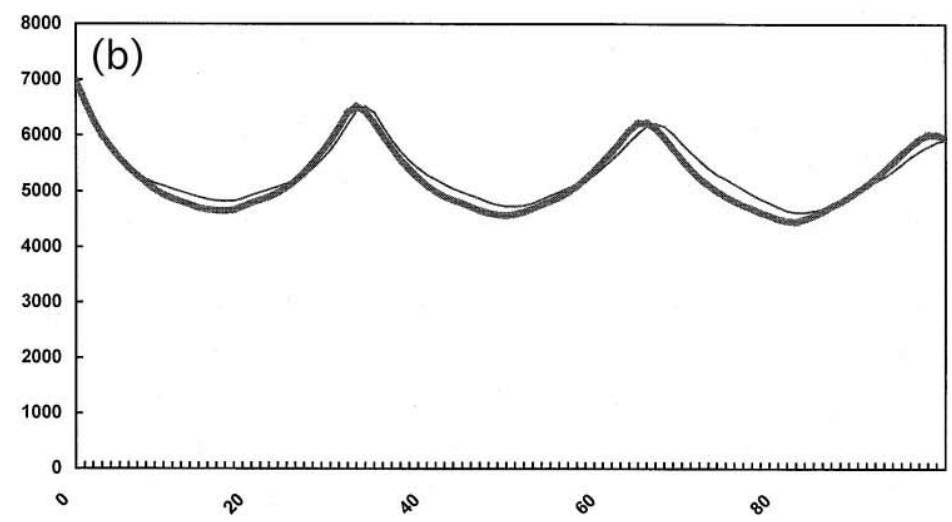

Fig. 3. (a) Texture image D102; (b) conditional covariance curve of example texture image D102 (the thin line presents the horizontal period, and the bold line the vertical period).

Fig. 3(a). In our experiments, the position of the first peak of the conditional covariance curve is taken as the period of the texture primitive.

\section{Texture primitive and window size.}

In a pixel classification application, the selection of a window size $W$ will affect the training results of a maximum likelihood classifier. Let us consider the situation mentioned in Section 2. If $W \leq r$, then the window $W$ may lie entirely in the ball of radius $r$ or $R$. Moreover, if the distance $d$ between disjoint balls is less than $W$, then $W$ may lie partly in balls of radius $r$ and $R$. Otherwise, $W$ may lie between disjoint balls. Thus, if one randomly chooses sample points from a texture image to compute the local granulometric size distributions associated with them, then the local pattern spectrum mean values will vary in the range from 0 to $R$, and the pattern spectrum standard deviation of this texture image is large. If $r<W \leq R$, the local pattern spectrum mean values vary from $r$ to $R$. Note that the probability of the event that $W$ lies between disjoint balls is decreased. As we keep enlarging the window size, the variation of local pattern spectrum mean values is further decreased.

In a practical application, we assume that texture images are structural texture images. That is, the images are defined by texture primitives which appear in near regular repetition spatial arrangements (Haralick, 1979). In this case, as we enlarge the size of window $W$, the variation of the local pattern spectrum mean for each image is decreased. The variation will be mitigated when the window size is larger than the sizes of the texture primitives, due to the periodicity property of structural texture images. Fig. 4 illustrates the relation between $W$ and the pattern spectrum deviation for texture image D20. The structuring elements used in this example are the same as those used in (Dougherty et al., 1992a). The $x$-axis denotes the window size, the $y$-axis denotes the standard deviation. If the texture image set is separable, then the small deviation indicates that the local pattern spectrum means highly concentrate on the global pattern spectrum mean of the texture image and the probability of inaccurate classification can be reduced. From Fig. 4, we observe that the local minimum deviation occurs when the window size $W$ equals 29. This is the same as the period of texture image D20. The variation of the local pattern spectrum means is mitigated whenever the size of window $W$ is larger than the period of repetition.

From another point of view, the maximum likelihood classifier is based on Bayes theory. Its simplest form is shown in Fig. 5. The shaded area denotes the probability of error for a maximum likelihood classification. We can observe that if the features of the 


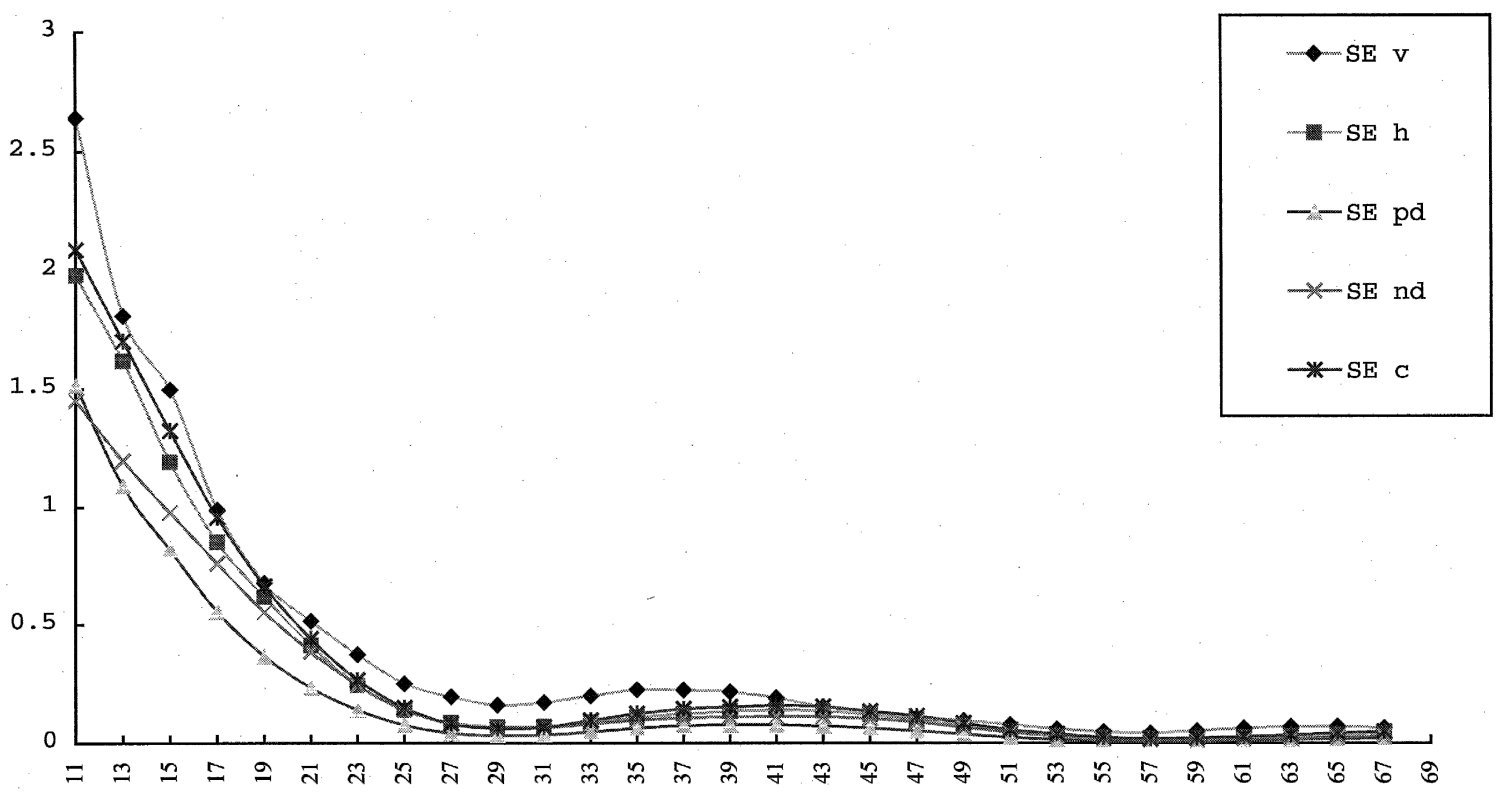

Fig. 4. Relation between window size $W$ and the pattern spectrum standard deviation of example texture image D20.

samples highly concentrate on the mean of the class, the probability of the error for the maximum likelihood classification is small. Hence, if the features of samples concentrate more on the mean of the class, a high accuracy rate can be obtained.

As mentioned in the previous discussion, the variation of the local pattern spectrum means of the features of the samples tend to mitigate whenever the window size is larger than the size of the texture primitive. The texture primitive can be found by investigating the variation of co-occurrence matrices or covariance. Once texture primitives for a set of texture images are found, the maximum size of the texture primitives is chosen as the window size. This window size will determine the local granulometric size distributions of this set of images. Let $T_{1}, T_{2}, \ldots, T_{n}$ be a set of texture images. Let $w_{1}, w_{2}, \ldots, w_{n}$ and $h_{1}, h_{2}, \ldots, h_{n}$ be periods of texture primitives along the $x$ - and $y$-axis, respectively. The window size of the local granulometric size distribu-

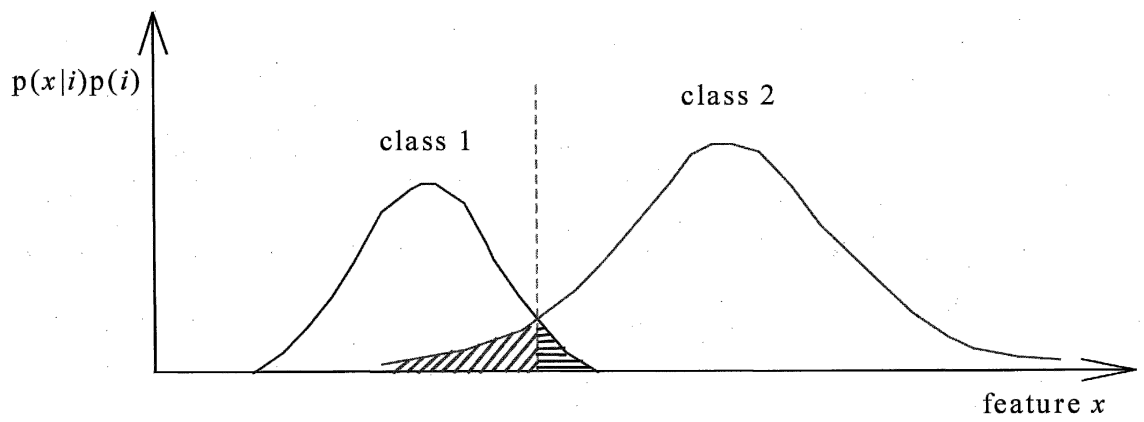

Fig. 5. A simplest example form for a maximum likelihood classification. 


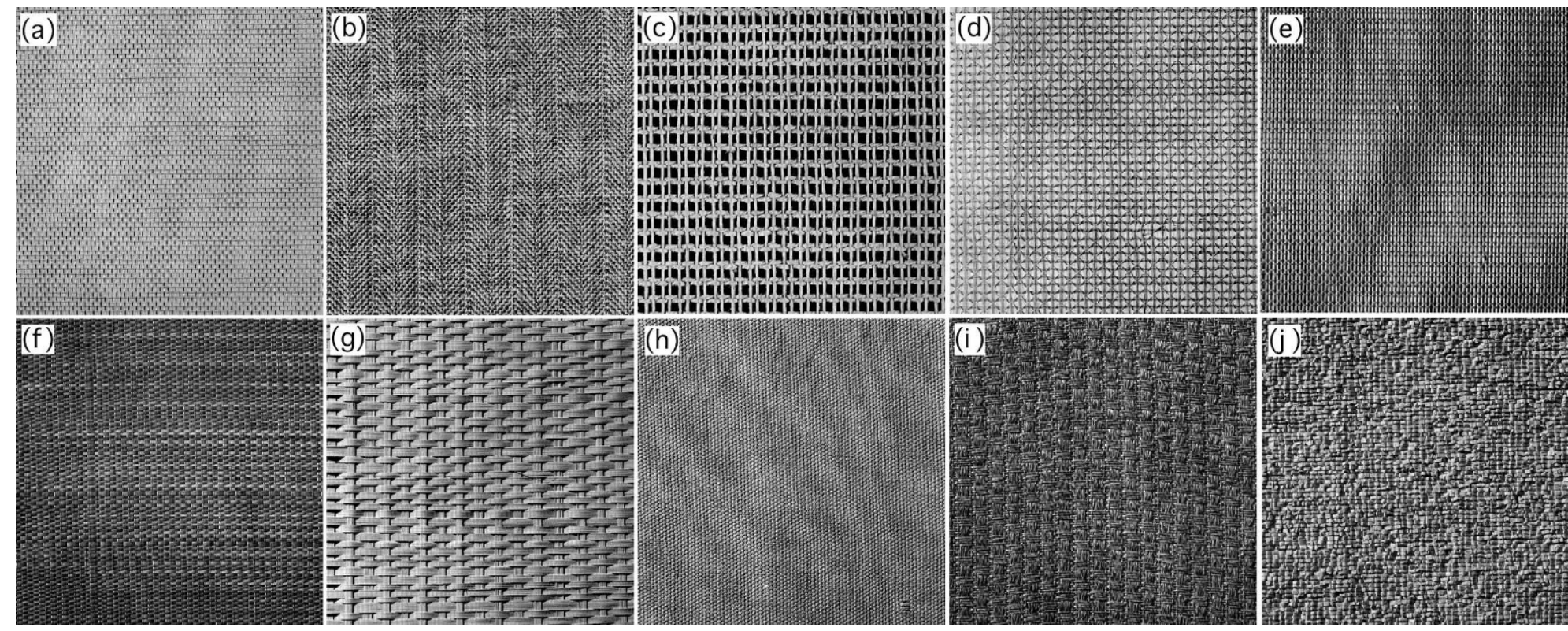

Fig. 6. Grey scale texture images. First row, left to right: (a) D6; (b) D17; (c) D20; (d) D52; (e) D53). Second row, left to right: (f) D55; (g) D65; (h) D77; (i) D82; (j) D84. 
Table 1

Texture primitive of original and thresholded image set

\begin{tabular}{|c|c|c|c|c|}
\hline \multirow[t]{2}{*}{ Texture image } & \multicolumn{2}{|c|}{ Grey scale } & \multicolumn{2}{|c|}{ Binary } \\
\hline & $x$-axis & $y$-axis & $x$-axis & $y$-axis \\
\hline d6 & 14 & 16 & 13 & 16 \\
\hline $\mathrm{d} 17$ & 10 & 11 & 10 & 11 \\
\hline $\mathrm{d} 20$ & 29 & 28 & 29 & 28 \\
\hline $\mathrm{d} 52$ & 13 & 26 & 15 & 25 \\
\hline d53 & 6 & 17 & 6 & 17 \\
\hline $\mathrm{d} 55$ & 19 & 8 & 19 & 8 \\
\hline d65 & 5 & 22 & 5 & 22 \\
\hline d77 & 11 & 6 & 11 & 6 \\
\hline $\mathrm{d} 82$ & 5 & 8 & 10 & 8 \\
\hline $\mathrm{d} 84$ & 10 & 14 & 10 & 5 \\
\hline
\end{tabular}

tion for this set of images is the maximal value of $w_{1}, w_{2}, \ldots, w_{n}$ and $h_{1}, h_{2}, \ldots, h_{n}$, i.e., window_size $=\max \left\{w_{1}, w_{2}, \ldots, w_{n}, h_{1}, h_{2}, \ldots, h_{n}\right\}$. It should be noted that the chosen window size is increased by one when it is an even number.

\section{Experimental results and discussion}

A great deal of data have been tested for the experiment. Ten test images are taken from Brodatz's collection of photographic texture images (Brodatz, 1966). A MicroTek ScanMarker 600Z scanner operating under Aldus Photostyler is used. The images are scanned at: $75 \mathrm{dpi}$ and $100 \%$ scaling into an 8-bit grey digital format. Fig. 6 shows the ten grey scale texture images (image set A). For the same reason as in mentioned by Dougherty et al. (1992a), a single threshold value is employed across all ten texture images. The moment preserving threshold method (Tsai, 1985) is chosen for binarization. The structuring element sequences we used are vertical (v), horizontal $(\mathrm{h}),+45^{\circ}$ diagonal $(\mathrm{pd}),-45^{\circ}$ diagonal (nd), and circular (c). The features used are the three central moments: the pattern-spectrum mean (PSM), standard deviation (PSSD), and skewness (PSS) for each structuring element sequence. Two additional features are also used: MAXLIN and LINEARLY. The classifier used in our experiment is the Gaussian maximum likelihood classifier, the same as the one reported by Dougherty et al. (1992a).

The effective window size has to be determined before performing granulometric classification. As mentioned previously, the effective window size can be determined by investigating the variation of covariance of each texture image. Table 1 shows the size of the texture primitives found in the threshold

Table 2

Relation of window size and accuracy rate in the original and threshold image set

\begin{tabular}{|c|c|c|c|c|}
\hline \multirow[t]{2}{*}{ Window size } & \multicolumn{2}{|l|}{ Test image set A } & \multicolumn{2}{|l|}{ Test image set B } \\
\hline & $\begin{array}{l}\text { Classification } \\
\text { accuracy } \\
(\%) \text { (grey scale) }\end{array}$ & $\begin{array}{l}\text { Classification } \\
\text { accuracy } \\
\text { (\%) (binary) }\end{array}$ & $\begin{array}{l}\text { Classification } \\
\text { accuracy } \\
(\%) \text { (grey scale) }\end{array}$ & $\begin{array}{l}\text { Classification } \\
\text { accuracy } \\
\text { (\%) (binary) }\end{array}$ \\
\hline 17 & 96.6 & 95.4 & 97.8 & 93.1 \\
\hline 19 & 97.1 & 96.1 & 98.0 & 95.2 \\
\hline 21 & 97.9 & 97.5 & 99.0 & 96.9 \\
\hline 23 & 98.7 & 97.6 & 99.6 & 97.1 \\
\hline 25 & 98.5 & 98.2 & 99.7 & 97.8 \\
\hline 27 & 99.2 & 98.2 & 99.9 & 98.1 \\
\hline 29 & 99.1 & 98.1 & 100 & 98.1 \\
\hline 31 & 99.5 & 98.3 & 100 & 98.4 \\
\hline 33 & 99.0 & 98.1 & 100 & 98.2 \\
\hline 35 & 99.1 & 98.4 & & 98.4 \\
\hline 37 & 99.3 & 98.2 & & 98.4 \\
\hline 39 & 98.9 & 98.3 & & 98.3 \\
\hline 41 & 99.1 & 98.5 & & 98.6 \\
\hline 43 & 99.1 & 98.7 & & 98.8 \\
\hline 45 & 99.4 & 98.8 & & 98.8 \\
\hline 47 & 99.6 & 98.9 & & 98.7 \\
\hline
\end{tabular}


images and the images without thresholding. In the threshold image set, the maximum primitive size, 29, is chosen as the window size for granulometric classification. Table 2 presents the relation between window size and accuracy rates of classification. The experimental results reveal that the selected window size yields a high accuracy rate. Table 2 also shows the relation of window sizes and accuracy rates for classification on the same image set without thresholding. The results confirm our idea again. The maximum primitive size is 29 as well. The results also indicate that classification with the window size predicted by the proposed method results in a high accuracy rate.

We also apply the proposed method to image set $\mathrm{B}$, which is the same as image set $\mathrm{A}$, except that image D55 is replaced by D102. In image D102, the texture primitive has size 33 and 32 along the $x$ - and $y$-axis, respectively. Size 33 is selected as the window size for granulometric classification with image set B. For the grey scale case, the results are very satisfying, all pixels are classified correctly. The relation between window sizes and accuracy rates is shown in the right portion of Table 2. Observe that the classification accuracy rate is already $100 \%$ when the selected window size is 29 .

Different test image sets need different window sizes to achieve effective classification. In general, larger windows decrease the variability of the feature at the cost of positional uncertainty. Given an input image set, the proposed method can predict a window size for granulometric classification. This window size can yield high classification accuracy and it is acceptably small. However, an explicit limitation of the proposed method is that the size of the largest texture primitive cannot be larger than that of the region to be classified. Otherwise, significant errors will occur.

\section{Conclusions}

We propose a method to determine the window size for local granulometric size distributions. The co-occurrence matrices and covariance measures are used to predict effective window sizes for granulometric classification. The proposed method suggests that one can choose the maximum size of texture primitives in an image set as the window size for local granulometric size distributions. Instead of the overall classification accuracy calculation, the use of the predicted window size can reduce the computation time. Moreover, the proposed method can automatically determine the effective window size when inputting a test image set.

\section{References}

Brodatz, P., 1966. Textures: A Photographic Album for Artists and Designers. Dover, New York.

Dougherty, E.R., Kraus, E.J., Pelz J.B., 1989. Image segmentation by local morphological granulometries. IGARSS'89, Vancouver, pp. 1220-1223.

Dougherty, E.R., Newell, J.T., Pelz, J.B., 1992a. Morphological texture-based maximum-likelihood pixel classification based on local granulometric moments. Pattern Recognition 25 (10), 1181-1198.

Dougherty, E.R., Pelz, J.B., Sand, F., Lent, A., 1992b. Morphological image segmentation by local granulometric size distributions. J. Elect. Imaging 1 (1), 46-60.

Dewaele, P., Van Gool, L., Wambacq P., Oosterlinck, A., 1988. Texture inspection with self-adaptive convolution filters. 9th ICPR, Rome, pp. 56-60.

Giardina, C.R., Dougherty E.R., 1988. Morphological Methods in Image and Signal Processing. Prentice-Hall, Englewood Cliffs, NJ.

Haralick, R.M., 1979. Statistical and structural approaches to texture. Proc. IEEE 67 (5), 786-804.

Matheron, G., 1975. Random Sets and Integral Geometry. Wiley, New York.

Parkkinen, J., Selkainaho, K., Oja, E., 1990. Detecting texture periodicity from the cooccurrence matrix. Pattern Recognition Letters 11, 43-50.

Serra, J., 1982. Image Analysis and Mathematical Morphology. Academic Press, London.

Starovoitov, V.V., Jeong, S.Y., Park, R.H., 1995. Investigation of texture periodicity extraction. SPIE 2501, 870-881.

Tsai, W.H., 1985. Moment preserving thresholding: a new approach. Computer Vision, Graphics, and Image Processing 29 (3), 377-393.

Zucker, S.W., Terzopoulos, D., 1980. Finding structure in co-occurrence matrices for texture analysis. Computer Graphics and Image Processing 12, 286-308 